\title{
Ultrasound-Guided Carpal Tunnel Release Using Dynamic Expansion of the Transverse Safe Zone in a Patient With Postpolio Syndrome: A Case Report
}

\author{
Troy Henning, DO, Daniel Lueders, MD, Kate Chang, Lynda Yang, MD
}

\begin{abstract}
The prevalence of carpal tunnel syndrome (CTS) in patients with postpolio syndrome occurs at a rate of $22 \%$. Irrespective of those with CTS, 74\% of postpolio patients weight bear through their arms for ambulation or transfers. As open carpal tunnel release is performed along the weight-bearing region of the wrist, their functional independence may be altered while recovering. This case demonstrates that ultrasound-guided carpal tunnel release was successfully performed in a patient with postpolio syndrome allowing him to immediately weight bear through his hands after the procedure so he could recover at home.

Level of Evidence: $\mathrm{V}$
\end{abstract}

\section{Introduction}

The prevalence of carpal tunnel syndrome in the general population is $0.6 \%-3.4 \%$ [1]. However, in patients with postpolio syndrome, the prevalence rate is $22 \%-62 \%$ [2]. For chronic cases, patients may elect to undergo surgical release [3]. Surgical releases include either open or endoscopic techniques, the latter being more controversial given its steeper learning curve [4]. Since 1997, more than 621 ultrasound-guided carpal tunnel releases (UGCTRs) using a variety of techniques have been published, with no reports of iatrogenic injuries [5-7]. This case demonstrates bilateral UGCTR using dynamic expansion of the transverse safe zone in a forearm crutch ambulator with postpolio syndrome.

\section{Case Presentation}

A 69-year-old man with postpolio syndrome and 5 years of carpal tunnel syndrome symptoms was referred for bilateral ultrasound-guided carpal tunnel release. He elected to decline open carpal tunnel release as he was still working and did not want to be burdened while recovering from bilateral releases in a nursing home. Physical examination revealed diminished light touch along the volar thumb through radial ring fingers bilaterally. Both sides demonstrated gross mild atrophy of the thenar muscles along with slight weakness of thumb abduction and flexion at the level of the carpometacarpal joint. Paresthesias were reproduced with a positive Tinel sign along the median nerve just proximal to the entrance of the carpal tunnel and with a positive Phalen maneuver at the wrists. He denied more proximal symptoms and had an absence of symptoms with Spurling maneuver bilaterally. Electrodiagnostic examination revealed right median sensory latency $4.8 \mathrm{~ms}$, left $5.4 \mathrm{~ms}$; right sensory amplitude $6.0 \mu \mathrm{V}$, left $4.1 \mu \mathrm{V}$; right median motor latency $5.2 \mathrm{~ms}$, left $5.6 \mathrm{~ms}$; and right amplitude $3.8 \mathrm{mV}$, left $3.2 \mathrm{mV}$. Needle examination revealed no spontaneous activity but polyphasic longduration units with increased amplitude and reduced recruitment in the opponens pollicis bilaterally. Sonographic cross-sectional area at the region of maximal enlargement just proximal to the right carpal tunnel was $15.8 \mathrm{~mm}^{2}$, left $17.2 \mathrm{~mm}^{2}$ (Table 1).

Before releases, the bilateral carpal tunnels were sonographically assessed to determine if the patient's anatomy would safely allow for ultrasound-guided release. This entailed measuring the transverse safe zone (TSZ; distance between the radial border of the hook of hamate or the ulnar artery, whichever is more radial, and the ulnar border of the median nerve). A TSZ greater than 0 is desirable (his left TSZ was $6.9 \mathrm{~mm}$, and the right TSZ was $6.6 \mathrm{~mm}$ ) as it increases the margin of safety between the knife and the ulnar neurovascular structures. The median recurrent branch to the thenar 
Table 1

Median nerve electrodiagnostic and sonographic image data

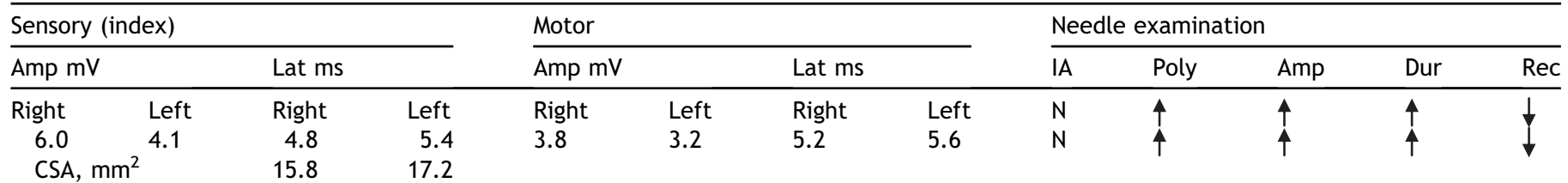

Amp = amplitude; Lat = latency; IA = insertional activity; Poly = polyphasic; Dur = duration; Rec = recruitment; CSA = cross-sectional area.

muscles was identified to ensure it arose from one of the radial or ventral aspects of its terminal divisions. He had no accessory or transligamentous branches. Based on his symptoms, electrodiagnostic testing, and no anomalous anatomic variants, he was deemed an appropriate candidate for the procedure.

He underwent a staggered bilateral UGCTR, left followed by right 1 month later. As this was a new technique for the provider and the institution, it was deemed prudent to perform the releases while the patient was anesthetized under monitored anesthesia care with a neurosurgeon (L.Y.) present in the room for backup if needed. Once the patient was anesthetized, the area was cleansed from the finger tips to the elbow with povoiodine and draped using sterile disposable drapes; no tourniquet was used. Using a 15-6 lineararray transducer (Sonosite X-porte, Bothell, WA), a 25 -gauge, 2 -in. needle was directly visualized anesthetizing the incision site and the subcutaneous palmar region with $10 \mathrm{~mL}$ of $1 \%$ lidocaine. A separate 25 -gauge, 2 -in. needle was visualized injecting $10 \mathrm{~mL}$ of sterile $0.9 \%$ sodium chloride solution into the carpal tunnel to help define the boundaries of the transverse carpal ligament, tendons, and median nerve. A 5-mm-long and approximately 4-5-mm-deep incision was made through the skin and antebrachial fascia with a No. 15 blade scalpel at the level of the proximal wrist crease, through which the device was introduced. The device was then advanced under direct sonographic guidance into the carpal tunnel until the tip was positioned just proximal to the palmar arch and fat pad and approximately $1 \mathrm{~cm}$ distal to the tapered end of the transverse carpal ligament (Figure 1). After confirming proper location in and out of plane relative to the transducer, the balloons within the device were inflated to widen the margin of safety between the knife and adjacent neurovascular structures (Figure 2). Then the knife was deployed to transect the transverse carpal ligament from a distal to proximal direction. Two passes of the knife were used on each side to ensure the ligament was completely released. Following release, the blunt tip of the device, with the balloons deflated and the knife recessed, was used to assess the completeness of release of the TCL (Figure 3). The wound was closed with a single 4-0 nylon suture on the left side and SteriStrip on the right, done at the discretion of the physician. Each time he was discharged home and able to immediately ambulate using the forearm crutches. Discharge instructions included to call or go to the emergency department for any increased pain, bleeding, swelling, fever, or new symptoms. He was to follow up in clinic in 1 week for suture removal and/or wound check. At his 1-week follow-up appointment, following each release, all incisions had healed, and the suture was removed on the left. He noted immediate relief of his nocturnal paresthesias at each of the initial follow-up visits. He reported near-complete resolution of daytime symptoms and was experiencing only mild discomfort along the palms when weight bearing through the hands.

To help track his symptoms and function in a more objective manner, 2 validated outcome measures were utilized. The Boston Carpal Tunnel Questionnaire (BCTQ) and the Shortened Disabilities of the Arm, Shoulder and Hand questionnaire (QuickDASH) scores were collected preoperatively and at 1 and 3 months after the releases. Both measures assess symptoms and function and are commonly used to assess treatment outcomes related to CTS $[8,9]$. Higher scores correspond with a greater degree of disability. The BCTQ is subdivided into a symptom score and functional status score; each has a calculated score ranging from 1-5. The QuickDASH is scored 0-100. Following open release, the minimal clinically important difference for changes in the BCTQ symptom and functional status scores are 1.14 and 0.74 , respectively [10]. The minimal clinically

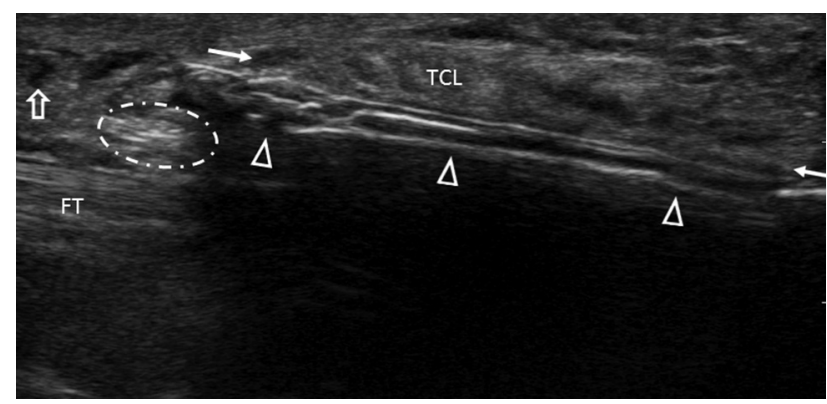

Figure 1. Sonographic view of the device in plane with the transducer. The transducer is in the sagittal plane. Note the device (open arrow heads) just deep to the transverse carpal ligament (TCL) (between closed arrows) and superficial to the flexor tendons (FT) and palmar fat pad (dashed oval). The palmar arterial arch is just above the open arrow. Top $=$ superficial; Bottom $=$ deep; Right $=$ proximal; Left $=$ distal. 


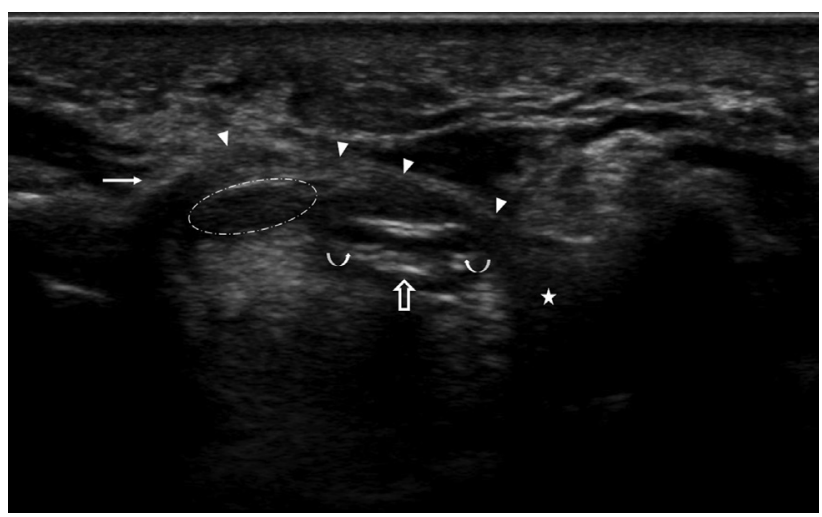

Figure 2. Short-axis view of the distal carpal tunnel with the device (open arrow) in place and balloons (curved arrows) deployed. The transducer is in the axial plane. Arrow = tubercle of trapezium; Star = hook of hamate; Dashed oval $=$ median nerve; Arrow heads $=\mathrm{TCL}$; Top $=$ superficial; Bottom $=$ deep; Right $=$ ulnar; Left $=$ radial.

important difference for the QuickDASH is a change greater than 20 [11]. After 12 weeks, his BCTQ symptom score improved by 2.0 (left) points and 1.62 (right). The functional score improved by 1.25 (left) and 1.37 (right). The QuickDASH score improved by 52.27 (left) points and 34.09 (right). The work module component of the QuickDASH form improved by 12 points after 12 weeks (Table 2). Two global outcome measures assessing if symptoms resolved (yes/no) and how satisfied he was with the results $(5$-point ordinal scale: $1=$ very dissatisfied, 2 = dissatisfied, $3=$ neither satisfied nor dissatisfied, $4=$ satisfied, $5=$ very satisfied) was recorded at 4 weeks after each release. He noted complete resolution of symptoms and being very satisfied with the results on both sides.

\section{Discussion}

Carpal tunnel syndrome is a very common disorder, resulting in more than 500000 surgeries performed in

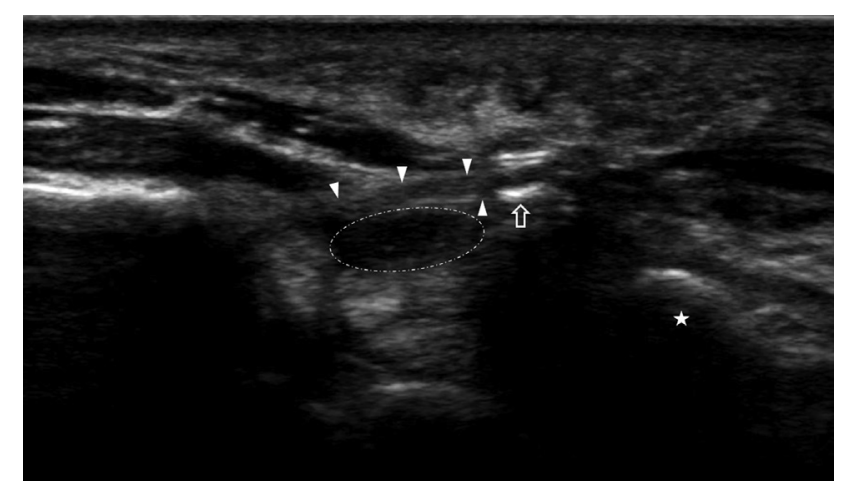

Figure 3. Short-axis view of the distal carpal tunnel postrelease. The transducer is in the axial plane. Note the device (open arrow) sitting superficial relative to the TCL (arrow heads) and hook of hamate (star). Dashed oval $=$ median nerve; Top $=$ superficial; Bottom $=$ deep; Right $=$ ulnar; Left $=$ radial.
Table 2

Boston Carpal Tunnel Questionnaire (BCTQ) and Shortened Disabilities of the Arm, Shoulder and Hand questionnaire (QuickDASH) results

\begin{tabular}{lllll}
\hline & & & & QuickDASH \\
& BCTQ (S) & BCTQ (FS) & QuickDASH & \begin{tabular}{l} 
(WM) \\
\hline Left side
\end{tabular} \\
$\quad$ & & & \\
$\quad$ Prerelease & 3.1 & 2.5 & 54.54 & 12 \\
4 wk & 1.1 & 1.25 & 15.91 & 6 \\
12 wk & 1.1 & 1.25 & 2.27 & 0 \\
$\quad \begin{array}{l}\text { pre-12 wk } \\
\text { Right side }\end{array}$ & 2.0 & 1.25 & 52.27 & 12 \\
$\quad$ Prerelease & 2.72 & 2.5 & 40.91 & 12 \\
4 wk & 1.27 & 1.25 & 4.54 & 6 \\
12 wk & 1.1 & 1.13 & 6.82 & 0 \\
$\Delta$ pre-12 wk & 1.62 & 1.37 & 34.09 & 12 \\
\hline
\end{tabular}

$\mathrm{S}=$ symptom score; $\mathrm{FS}=$ functional status score; $\mathrm{WM}=$ work module; $\Delta$ pre-12 $\mathrm{wk}=$ difference between prerelease and 12-wk scores.

the United States annually [7]. Although the traditional open or endoscopic techniques may work for the majority, it may be challenging for those who use their hands for transfers, ambulation or other self-care needs. Our case demonstrates that ultrasound-guided carpal tunnel release using dynamic expansion of the TSZ can be performed in a safe and effective manner in a patient with postpolio syndrome without interfering with his or her independence. The patient was able to go home the same day using his forearm crutches and reported immediate resolution of nocturnal symptoms. At 3 months postrelease, his improvements in the BCTQ and QuickDASH scores are similar to those reported 3-6 months following both open and endoscopic release. Atroshi et al compared endoscopic to open release and noted that both groups showed improvements at 3 months in the BCTQ symptom and functional status scores of 1.5 and 1.3, respectively [12]. Similarly, other studies assessing endoscopic and open surgeries showed improvements in DASH scores of $13-20$ points by 6 months $[13,14]$.

Since this case, we have been able to transition the releases to an outpatient procedural center under local anesthesia only with similar results and excellent patient tolerance. Along with this comes increased convenience for patients. Specifically, (1) patients are not asked to take nothing by mouth prior to the procedure, (2) there is reduced waiting/procedure time on the day of the release, and (3) parking is often easier at an outpatient clinic setting. Additionally, there are potential cost savings associated with this approach. The estimated cost of USCTR is $\$ 814$, including 1 postoperative follow-up visit. According to other recent studies, open and endoscopic release are estimated to cost $\$ 1310-\$ 2160$, respectively $[15,16]$.

We acknowledge that some will have safety concerns with nontraditional surgeons performing this procedure. However, a simple review of the literature reveals that more than 600 ultrasound-guided carpal tunnel releases have been performed by a variety of specialists with no 
reports of injuries $[5-7,17]$. This iatrogenic injury rate is lower than what is reported as it relates to open $(0.49 \%)$ and endoscopic $(0.19 \%)$ releases [4]. Potential explanations are that (1) high-frequency transducers have a spatial resolution of $150 \mu \mathrm{m}$, allowing for all the relevant anatomy to be visualized, further enhancing the margin of safety [18], and (2) smaller instruments reduced the risk of nerve compression as maybe seen with endoscopic release [4]. Interventional physiatrists are quickly becoming the content experts with regard to interventional ultrasonographic procedures and as a result are the ones driving the advancements in the field. Those with the appropriate level of training and skill set will be suited to perform these procedures. In conclusion, this minimally invasive method of carpal tunnel release appears to offer significant advantages over traditional release to patients who rely on their upper extremities for transfers and ambulation.

\section{References}

1. de Krom MC, Knipschild PG, Kester AD, Thijs CT, Boekkooi PF, Spaans F. Carpal tunnel syndrome: Prevalence in the general population. J Clin Epidemiol 1992;45:373-376.

2. Werner R, Waring D. Risk factors for median mononeuropathy of the wrist in postpoliomyelitis patients. Arch Phys Med Rehabil 1989;70:464-467.

3. Huisstede BM, Hoogvliet P, Randsdorp MS, Glerum S, van Middelkoop M, Koes BW. Carpal tunnel syndrome. Part I: effectiveness of nonsurgical treatments-a systematic review. Arch Phys Med Rehabil 2010;91:981-1004.

4. Benson LS, Bare AA, Nagle DJ, Harder VS, Williams CS, Visotsky JL. Complications of endoscopic and open carpal tunnel release. Arthroscopy 2006;22:919-924, 924.e1-924.e12.

5. Guo D, Tang Y, Ji Y, Sun T, Guo J, Guo D. A non-scalpel technique for minimally invasive surgery: percutaneously looped thread transection of the transverse carpal ligament. Hand (N Y) 2015;10: 40-48.
6. Petrover D, Silvera J, De Baere T, Vigan M, Hakimé A. Percutaneous ultrasound-guided carpal tunnel release: Study upon clinical efficacy and safety. Cardiovasc Intervent Radiol 2016;40:568-575.

7. Rojo-Manaute JM, Capa-Grasa A, Chana-Rodriguez F, et al. Ultra-minimally invasive ultrasound guided carpal tunnel release: a randomized controlled trial. J Ultrasound Med 2016;35: $1149-1157$.

8. Greenslade JR, Mehta RL, Belward P, Warwick DJ. Dash and Boston questionnaire assessment of carpal tunnel syndrome outcome: What is the responsiveness of an outcome questionnaire? J Hand Surg Br 2004;29:159-164.

9. Gummesson C, Ward MM, Atroshi I. The shortened Disabilities of the Arm, Shoulder and Hand questionnaire (QuickDASH): Validity and reliability based on responses within the full-length DASH. BMC Musculoskelet Disord 2006;7:1-7.

10. Kim JK, Jeon SH. Minimal clinically important differences in the Carpal Tunnel Questionnaire after carpal tunnel release. J Hand Surg Eur Vol 2012;38:75-79.

11. Clement ND, Duckworth AD, Jenkins PJ, McEachan JE. Interpretation of the QuickDASH score after open carpal tunnel decompression: Threshold values associated with patient satisfaction. J Hand Surg Eur Vol 2016;41:624-631.

12. Atroshi I, Larsson G-U, Ornstein E, Hofer M, Johnsson R, Ranstam J. Outcomes of endoscopic surgery compared with open surgery for carpal tunnel syndrome among employed patients: Randomised controlled trial. BMJ 2006;332:1473.

13. Kotsis SV, Chung KC. Responsiveness of the Michigan Hand Outcomes Questionnaire and the Disabilities of the Arm, Shoulder and Hand questionnaire in carpal tunnel surgery. J Hand Surg Am 2005; 30:81-86.

14. Weber RA, Rude MJ. Clinical outcomes of carpal tunnel release in patients 65 and older. J Hand Surg Am 2005;30:75-80.

15. Park KW, Dickerson C. Can efficient supply management in the operating room save millions? Curr Opin Anaesthesiol 2009;22: 242-248.

16. Macario A. What does one minute of operating room time cost? J Clin Anesth 2010;22:233-236.

17. Buncke G, McCormack B, Bodor M. Ultrasound-guided carpal tunnel release using the manos CTR system. Microsurgery 2013;33: 362-366.

18. Nazarian LN. The top 10 reasons musculoskeletal sonography is an important complementary or alternative technique to MRI. AJR Am J Roentgenol 2008;190:1621-1626.

\section{Disclosure}

T.H. Swedish Medical Group, Swedish Spine, Sports and Musculoskeletal Medicine, 1600 E. Jefferson Street, Suite 300, Seattle, WA 98122. Address correspondence to: T.H.; e-mail: troy.psu@gmail.com

Disclosure: nothing to disclose

D.L. Department of Physical Medicine and Rehabilitation, University of Michigan, Ann Arbor, MI

Disclosure: nothing to disclose
K.C. Department of Neurosurgery, University of Michigan, Ann Arbor, MI Disclosure: nothing to disclose

L.Y. Department of Neurosurgery, University of Michigan, Ann Arbor, MI Disclosure: nothing to disclose

Submitted for publication July 3, 2017; accepted February 27, 2018. 